\title{
Valuing Flexibility in the Migration to Flexgrid Networks
}

\author{
Mathieu Tahon ${ }^{1}$, Sofie Verbrugge ${ }^{1}$, Didier Colle ${ }^{1}$, Mario Pickavet ${ }^{1}$, Paul Wright ${ }^{2}$, Andrew Lord ${ }^{2}$ \\ ${ }^{1}$ Ghent University-iMinds, Gaston Crommenlaan 8b201, 9050 Ghent, Belgium \\ ${ }^{2}$ BT Polaris House, Adastral Park, Martlesham Heath, Ipswich, United Kingdom, IP5 3RE \\ Mathieu.tahon@intec.ugent.be
}

\begin{abstract}
Flexible optical networking can now be installed to increase network capacity in light of future traffic demands. This paper researches the different migration paths using a real option analysis, showing the impact of uncertainty.

OCIS codes: (060.0060) Fiber optics and optical communications; (060.4250) Networks
\end{abstract}

\section{Future evolutions in telecom core networks}

With estimated exponential core network traffic growths due to increasing access network traffic, future core networks will need to increase their capacity. The channel capacity will need to be increased beyond 100G per channel or higher, together with an increase of spectral efficiency. Additionally, the dynamic functionality of networks should be increased, allowing for dynamic re-optimization.

To achieve the goals stated above, spectral flexibility - or flexgrid - has been identified as the key type of expected flexibility within dense wavelength division multiplexing (DWDM) systems. Present day DWDM systems operate under the Fixed Grid - or ITU grid. Multiplexers, optical network nodes and transponders are all tuned to this grid. The core network is based on IP offloading, transponders with fixed bit rate and modulation format. But traffic growth would require the use of several 10G or 40G channels between adjacent IP routers.

Flexgrid, making the wavelength switched optical network (WSON) elastic by moving away from the ITU grid, can accommodate both sub- and super wavelength traffic. It allows an adjustable use of optical spectrum within a certain granularity. This granularity is the minimum bandwidth slot that can be switched in the optical spectrum (6.25 and $12.5 \mathrm{GHz}$ are possible) [1]. This technology is slowly coming available, and operators have to decide whether or not to install this flexgrid capable equipment in their network. However, since this technology is still in its infancy, an installation today will be expensive or risk poor reliability. Additionally, the performance of this equipment might still be lacking the full functionality that may appear later.

In order to make the correct investment decision, a techno-economic analysis is conducted for the future installations in the UK core network, as described in [2]. Additionally, it is indicated how uncertainty surrounding the future conditions, e.g. traffic growth and equipment prices, influences the optimal migration path. Since the operator has the opportunity to alter this path according to the future conditions, this analysis is well suited for a real option analysis (ROA) [3]. First, the different migration paths will be introduced and a standard techno-economic analysis will be performed. Secondly, the ROA will be conducted to indicate the importance of flexibility and how it influences the investment decision.

\section{Different future migration options}

The need for flexgrid installations in the future core (and metro) networks will be mainly driven by the future traffic growth evolutions. Three different possible migration scenarios are described in Figure 1. In case of low traffic growth, the existing Fixed Grid installations might still suffice to cover traffic needs in the nearby future. When the implementation of flexgrid capable networks can be postponed for some years, the operator can take advantage of the cheaper installation of existing Fixed Grid DWDM technology. If traffic follows the expected growth path, this is the preferred solution. However, if it grows faster than expected, the operator will end up either losing money, since he is not able to route all traffic, or he will be putting in a next generation DWDM network -presumably flexgrid this time- earlier than he would have needed to, also leading to additional costs (DWDM migrate scenario).

The second option for operators is installing a full flexgrid ready network now, both equipment and muxponders (Flexgrid scenario). The advantages of such an installation are clear, under any traffic growth, the operator is ready for it. However, under low traffic growth, a lot of money will be spent in vain, since the network equipment is overqualified for the required traffic. Additionally, as was already indicated, the performance of current flexgrid equipment might considerably improve during the following years. As the learning curve is followed, this will also result in a decrease of the cost for such equipment. 


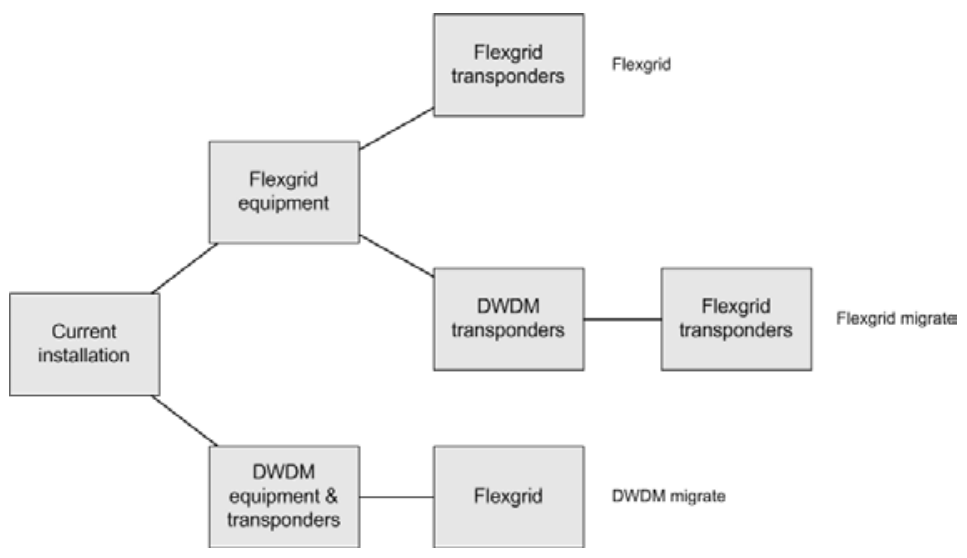

Fig. 1. Migration options towards flexgrid

There exists however an intermediate installation path which offers future flexibility for the operator (Flexgrid migrate scenario). By installing flexgrid capable equipment, but using standard DWDM transponders instead of flexgrid capable transponders, the operator buys the option for a cheaper migration towards flexgrid if necessary. Additionally, since the equipment is used with standard DWDM transponders, the performance risk and initial capital expenditure are decreased.

\section{Migration of the UK core network}

For each node of the reference network, a dimensioning is performed based on the expected traffic growth (37\% p.a.) and the cost input from the STRONGEST CapEx model [4]. The three migration scenarios described above are modeled. This migration is only performed when traffic growth in the next five years exceeds $300 \%$, which is in line with the current capacity limits of the UK core network. Under the basic assumption of $37 \%$ growth p.a., this threshold is not exceeded within the evaluation period of five years.

Based on the traffic estimations for each edge, the number of required muxponders is calculated for every year. For example, a link with 30Gbps will result in the installation of a 40G (line rate), 4X10G (client requirements), $2500 \mathrm{~km}$ (reach) muxponder. Traffic of 120Gbps requires one 100G, 10X10G, 2000km and one 40G, 4X10G, $2500 \mathrm{~km}$ muxponder. With $400 \mathrm{G}$ muxponders only becoming available later during the project lifetime, these can only be installed from that year. This means that once 400G muxponders are available, a link which initially required four 100G muxponders will then have a 400G, 10X40G, 150km muxponder. Of course, this installation can only be made if the reach constraint is satisfied. This calculation results in a number of muxponders required on the start and end node of every edge. The nodes are also modeled, with two parameters impacting the node cost, node degree and the number of wavelengths transported through the node. Currently standard nodes are used, so the nodes are not contentionless, colorless or directionless [5].

Once the number of nodes and muxponders is modeled, the CapEx evaluation of the different scenarios is performed. This is a straightforward exercise, where the amounts of necessary equipment are multiplied with their corresponding cost. Notice that for DWDM and flexgrid equipment, a cost erosion factor is included. For DWDM and flexgrid, this cost erosion is currently estimated at $10 \%$. These numbers are based on the extended learning curve [6]. The risk related to flexgrid equipment is modeled using a higher replacement need. To allow for a fair comparison of the different migration paths, an operational cost for energy has been added to the model. Since flexgrid is expected to reduce the required energy, the energy use of different muxponders is included.

\section{Evaluation of the migration scenarios}

It was already indicated that under the basic assumptions, no migration towards flexgrid would be required, and this is also reflected in the results of the techno-economic analysis of the three migration scenarios under the standard conditions. In Table 1, the total cost (in Strongest Cost Units) can be found for the three scenarios. The DWDM migrate scenario is clearly the most cost effective.

However, as was already indicated, the expected traffic growth is uncertain. This uncertainty might severely impact the total cost for each installation. Under high traffic growth, it might be more cost effective to already migrate to flexgrid instead of continuing to use standard DWDM Fixed Grid equipment. The limits of the latter would be reached faster, requiring a fast re-installation of new equipment. To assess the impact of uncertainty, a sensitivity analysis was performed. Five thousand simulations were performed, with a triangular uncertainty 
distribution on the traffic growth ranging between 30 and $70 \%$, with $37 \%$ as the most likely. The impact on the cost result is shown in Table 2.

Table 1. Total cost for the three migration scenarios

\begin{tabular}{|l|c|c|c|}
\hline & DWDM migrate & Flexgrid & Flexgrid migrate \\
\hline Cumulative discounted cost & $2.513 \mathrm{SCU}$ & $4.118 \mathrm{SCU}$ & $2.656 \mathrm{SCU}$ \\
\hline
\end{tabular}

Table 2. Impact of uncertain traffic growth on the cost for the migration strategies

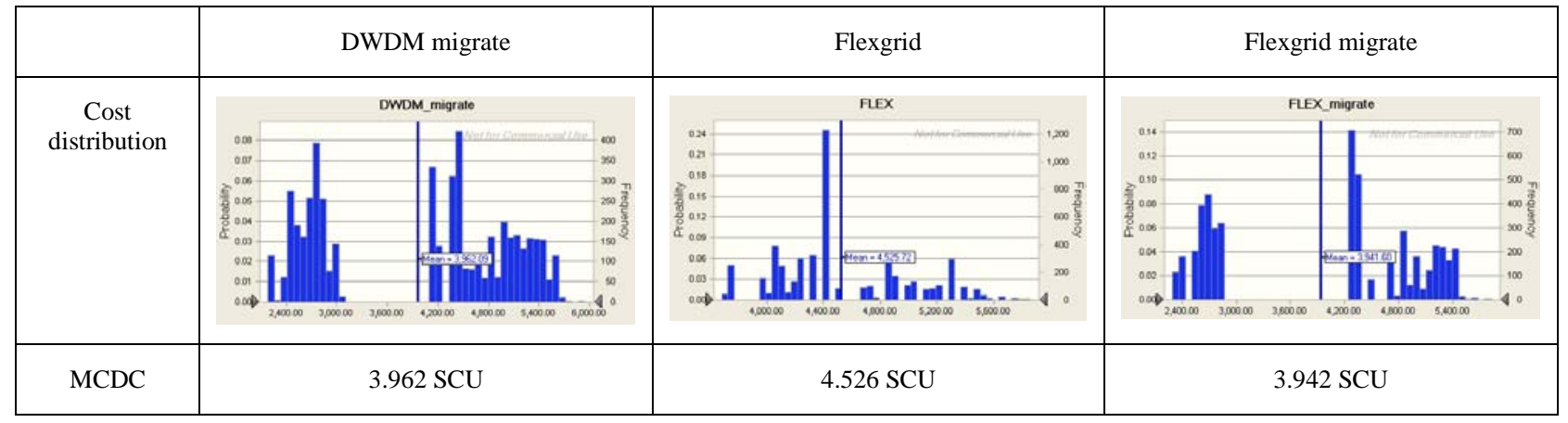

From the cost distributions, it is clear that the traffic uncertainty has a major impact on the cost evaluation of all three scenarios. For the DWDM migrate case, high traffic growth results in a large increase in total costs, as can be seen from the two peaks in the distribution. The same goes for the flexgrid migrate case, but the second peak has a shorter tail. This results in a lower mean cumulative discounted cost (MCDC) for the flexgrid migrate case compared to the DWDM migrate case. Buying initial flexibility at the start of the project, by installing more expensive, but flexgrid capable equipment, pays off. While in the analysis without uncertainty, the DWDM migrate scenario was still $5.6 \%$ more expensive, the impact of flexibility impacts this cost difference to a $0.5 \%$ cheaper flexible installation.

\section{Conclusions}

With ever increasing bandwidth demands in access networks, the traffic needs in metro and core networks will also increase. In order to cope with these needs, the installation of flexgrid capable nodes and transponders will become necessary in the future. Currently, this technology is still in its infancy, and a full installation now comes with a high degree of risk.

In this paper, different migration paths towards flexgrid were investigated under uncertainty, showing the value of flexibility of a gradual migration towards full flexgrid networks, which impacts the migration strategy to favor a flexible installation.

\section{Acknowledgement}

The research leading to these results has received funding from the European Community's Seventh Framework Programme FP7/2007-2013 under grant agreement n² 247674 (STRONGEST project).

\section{References}

[1] Spectral grids for WDM applications: DWDM frequency grid, ITU-T Recommendation G.694.1, (pre-published, 2012)

[2] Efficient and optimized network architecture: Requirements and reference scenarios, STRONGEST D2.1

[3] Copeland, T. E., \& Keenan, P. T. “How much is flexibility worth?” McKinsey Quarterly, (2), 38-50 (1998).

[4] Network planning/dimensioning/optimization strategies and mechanisms for network operation, STRONGEST D2.3

[5] S. Gringeri et al., "Flexible architectures for optical transport nodes and networks," IEEE Communications Magazine 48 (7), $40-50$ (2010).

[6] B.T. Olsen and K. Stordahl, “Models for forecasting cost evolution of components and technologies”, Telektronikk 4.04, pp. 138-148, 2004. 